\title{
Unearthing Faculty and Trainee Perspectives of Feedback in Internal Medicine: the Oral Case Presentation as a Model
}

\author{
James Rassos, $\mathrm{MD}^{7}$, Lindsay J. Melvin, $\mathrm{MD}^{2}$, Daniel Panisko, MD, MPH², \\ Kulamakan Kulasegaram, $P h D^{3}$, and Ayelet Kuper, $M D, P h D^{4,5,6}$
}

'General Internal Medicine, University of Toronto, Toronto, Ontario, Canada; ${ }^{2}$ Division of General Internal Medicine, University Health Network and Faculty of Medicine, University of Toronto, Toronto, Ontario, Canada; ${ }^{3}$ Faculty of Medicine, University of Toronto, Toronto, Ontario, Canada; ${ }^{4}$ Department of Medicine, Faculty of Medicine, University of Toronto, Toronto, Ontario, Canada; ${ }^{5}$ Wilson Centre for Research in Education, University Health Network/University of Toronto, Toronto, Ontario, Canada; ${ }^{6}$ Division of General Internal Medicine, Sunnybrook Health Sciences Centre, Toronto, Ontario, Canada.

BACKGROUND: The case presentation is a fundamental activity used in both patient care and trainee education, partly due to feedback from supervisor to trainee. Although feedback in medical education is well studied, prior studies have not focused on the perceptions of feedback by Internal Medicine supervisors and trainees as it relates to clinical activities like the case presentation.

METHODS: Semi-structured interviews were conducted with eight Internal Medicine physicians, and 18 Internal Medicine trainees (5 medical students, 13 residents) at the University of Toronto. Purposive sampling was used. Interviews were conducted and coded iteratively within a constructivist grounded theory approach until saturation was reached.

RESULTS: Supervisors and trainees recognized feedback as an important part of the case presentation that can be (1) explicit, labeled feedback or (2) implicit, unlabeled feedback. Both trainees and supervisors perceived that not enough feedback occurs, likely stemming from a hesitancy by supervisors to label implicit feedback, calling it an interruption instead. Although trainees were keenly aware of non-verbal feedback from their supervisors as implicit feedback, they often interpreted explicit constructive feedback negatively. Interestingly, the same feedback from senior residents was regarded as highly educational, as it was uncoupled from assessment.

CONCLUSION: Feedback occurs more frequently in case presentations than previously described, particularly in an implicit, unlabeled format. Even though under-recognized, trainees identify and utilize implicit feedback from supervisors, and coaching from senior residents, to develop learned behaviors. This is reassuring in the age of CompetencyBased Medical Education, as feedback has an essential role in workplace-based assessment and promotion.

$\overline{\text { Previous Presentations Faculty perceptions were presented at the }}$ International Conference on Residency Education and Canadian Society of Internal Medicine in 2016, and trainee perceptions were presented at the International Conference on Residency Education and Canadian Society of Internal Medicine in 2017.

Electronic supplementary material The online version of this article (https://doi.org/10.1007/s11606-019-05134-z) contains supplementary material, which is available to authorized users.

Received August 27, 2018

Revised February 5, 2019

Accepted May 21, 2019

Published online August 6, 2019
KEY WORDS: medical education; assessment and evaluation; postgraduate/undergraduate education.

$\mathrm{J}$ Gen Intern Med 34(10):2107-13

DOI: $10.1007 / \mathrm{s} 11606-019-05134-\mathrm{Z}$

(c) Society of General Internal Medicine 2019

\section{INTRODUCTION}

Feedback in medical education has long been recognized as an important activity to support student learning and development. ${ }^{1,2}$ However, in many medical education contexts, learners report that they rarely receive feedback, ${ }^{3,}{ }^{4}$ which the literature suggests may stem from the difficulty in recognizing the various forms it takes within medical education and the workplace. ${ }^{2,3}$ When learners and supervisors hear the word feedback, they often imagine an explicit, labeled event occurring as a planned activity at a predetermined time, such as at the end of a rotation or case presentation. ${ }^{5}$ However, feedback can also be implicit or unlabeled; it can occur "on the go" ${ }^{\prime 6}$ and may even take the form of role modeling important clinical activities. ${ }^{7}$ Feedback can be defined as the act of providing specific information to a trainee that compares their performance to a set standard in order to help them improve ${ }^{3}$; however, as this definition and others does not distinguish between types of feedback, it becomes challenging for supervisors and learners to recognize what "counts."

Further adding to the complexity is the introduction of Competency-Based Medical Education (CBME). CBME is an outcome-based approach to the curriculum design of medical education programs that aims to graduate trainees based on when they achieve competence, rather than when they fulfill a specified time in training. ${ }^{8}$ As many programs move forward with CBME, meaningful, formative feedback, divorced from summative assessment and embedded in clinical practice, will have a decisive role in meeting the promise of competencybased decisions. ${ }^{9}$ Thus, within any program adherent to a CBME framework, understanding the role of feedback in and around essential acts of daily clinical practice is essential. ${ }^{10}$

The case presentation, in which a patient's full history, physical examination, diagnosis, and management plan are formulated and delivered by trainee to supervisor, is an ideal 
context in which to study feedback. In internal medicine specifically, case presentations are a fundamental activity in patient care, ${ }^{11}$ with an opportunity for trainee education and learning through delivery of feedback. ${ }^{12}$ In fact, case presentations are crucial to medical education and practice and are included as milestones in both the USA and Canada. ${ }^{13-15}$

Apart from studies identifying shared expectations of trainee case presentations, ${ }^{12,}{ }^{16}$ there is a paucity of literature specifically exploring the relationship between feedback and case presentations as a routine workplace-based activity within internal medicine. As robust, ongoing feedback will be essential for the success of CBME, ${ }^{9}$ it is important to harness the potential of clinical activities such as the case presentation. Specifically, understanding how the case presentation fits within the feedback culture ${ }^{17,18}$ is crucial for optimizing their use in trainee education. Our study aims to explore how Internal Medicine clinical supervisors and trainees perceive feedback within the context of the case presentation.

\section{METHODS}

\section{Design}

We employed a constructivist grounded theory approach to explore the relationship between feedback and the case presentation. ${ }^{19,20}$ This study was part of a larger study understanding the use of case presentations in trainee medical education and assessment. ${ }^{21}$

\section{Study Population}

Our study population included University-Appointed Internal Medicine faculty supervisors of varying levels of experience at a large quaternary, multi-site academic center in downtown Toronto who routinely attend on Clinical Teaching Units, as well as Internal Medicine trainees and senior medical students who rotated through Internal Medicine rotations. In total, eight faculty members and 18 trainees (five medical students, five PGY1s, three PGY2s, and five PGY3s) were interviewed.

Study authors recruited participants via purposive email, until saturation was reached, and information was provided that explained the study in neutral terms. Face-to-face interviews were then arranged with interested parties. Research ethics approval was obtained from the University Health Network Research Ethics Board.

Purposive sampling of physicians was utilized to ensure heterogeneity in demographic variables as well as a range of possibly held opinions so that a thorough understanding of the case presentations could be obtained.

\section{Setting}

As with many other institutions, case presentations are a routine part of the morning intake of patients. Medical students and residents present admitted cases to their supervisors. Case presentations are not individually given a formal specific assessment and feedback is not a mandatory component of the case presentation review.

\section{Data Collection}

With informed written consent, study authors JR and LM conducted semi-structured interviews, which lasted 30-45 minutes. The interview guide included open-ended questions about the perception of the case presentation in trainee education and assessment, as well as questions specifically pertaining to feedback. All interviews were recorded and transcribed verbatim by study authors JR and LM. Consistent with grounded theory methodology, interviews and data analysis were performed contemporaneously, in an iterative, constant comparative approach. Continuous revisions were made to the interview guide to further explore concepts and themes.

\section{Data Analysis}

Data were analyzed using a constructivist grounded theory approach. ${ }^{22,}{ }^{23}$ We performed an iterative, constant comparative analysis to elicit recurring categories and themes. As this was part of a larger study examining the case presentation more globally, data analysis was conducted separately. After interviews were transcribed, study authors JR, LM, and DP completed line-by-line coding to develop initial codes and themes. The full research team, composed of a variety of individuals with unique educational lenses, contributed to the analysis. JR was a third-year Internal Medicine resident immersed in clinical work; LM is a newly graduated internist who has recently transitioned from presenting case presentations to solely reviewing them; $\mathrm{KK}$ is a scientist with expertise in assessment in medical education; AK is a mid-career IM specialist and clinical supervisor who is also an expert in qualitative methodology; DP is a senior IM specialist and educational administrator well known as an expert clinical teacher. After the first four interviews, the research team met to identify emerging concepts and to adapt the interview guide to address these concepts so they could be explored in more detail. JR, LM, and DP met twice more to finalize categories and explore the relationships between major themes in ongoing consultation with the rest of the team. Data collection continued until no novel themes or concepts arose, and theoretical saturation was reached. Theoretical saturation was determined to be the point in which sufficient data had been collected to enable a thorough understanding of the key concepts being explored. ${ }^{24,}{ }^{25}$ A separate analysis, led by coauthor LM, was also completed and has been submitted as its own, unique manuscript. ${ }^{21}$

\section{RESULTS}

Eight faculty members were interviewed with teaching experience ranging from 2 to 28 years. Eighteen trainees, of varying levels of training, were also interviewed. Quotations were identified by anonymous source codes. 
Both supervisors and trainees identified feedback as an integral part of day-to-day clinical activities on the Clinical Teaching Unit. As the case presentation is a routine workplace-based activity that "is one of the few opportunities for direct [...] observation and feedback" (faculty 8), it was used as a means to explore ways in which feedback is operationalized in Internal Medicine. Both trainees and supervisors also identified the case presentation as a time for assessment, and this topic was analyzed and presented in a separate manuscript. ${ }^{21}$

Although the majority of faculty members felt they provided feedback routinely during the case presentation, many acknowledged there was "...an issue in terms of feedback: we aren't able to give enough" (faculty 1 ). Trainees perceived a lack of feedback and desired more:

"I think that there should be feedback and it probably doesn't happen enough. There are always ways that whoever is presenting [ ... ] can get some information about how it can be improved." (trainee 18 (PGY3))

\section{Types of Feedback}

Despite the universal perception that feedback is scarce, ${ }^{3,4}$ interviews revealed that feedback does occur either as labeled, explicit feedback or as implicit, unlabeled feedback. Even more, supervisors and trainees identified the concepts they are receiving feedback on (Table 1), which may take the form of implicit or explicit feedback.

\section{Explicit Feedback}

Many supervisors noted the importance of explicitly labeling feedback to ensure learners are aware it is occurring. One senior faculty member explained "...often I am [labeling it] because we know that's important [that] we do." (faculty 8 ) Learners recognize explicit feedback easily, usually because it is identified as such and occurs at a set time, either at a midterm or end of rotation evaluation. Trainees noted that these explicit feedback moments more often focused on their overall performance as these sessions are usually removed from the case presentation itself. They also focused on how earlier feedback was incorporated into their clinical practice. As one trainee said:

"[Explicit feedback] is usually on overall performance: how you functioned as a member of the team, how you functioned as $[\ldots]$ teacher to the medical students, $[\ldots$ ], how in general your communication style works ... how reliable your impressions are, and how safe you are with patients overnight." (trainee 10 (PGY1))Though trainees perceive a lack of frequent feedback from supervisors, they highly value the more common explicit, constructive feedback from their senior residents. Trainees actually identified the coaching and feedback received from senior residents overnight as one of the most important educational components of the case presentation and came to expect this explicit feedback. The senior resident is a role model, teacher, and confidant for more junior learners, and most trainees interviewed described this relationship as the key cog within this environment.

"I don't think in the morning the presentation was in itself the most educational part for me, I think the most educational part was presenting to my senior resident in the evening..." (trainee 1 (medical student))Senior residents felt it was their duty to provide feedback on junior learners' case presentations, to prepare them for the presentation to supervisors in the morning, recognizing the morning presentation is higher stress and stakes:

Table 1 Topics of Feedback

\begin{tabular}{|c|c|c|c|}
\hline Element & Description & Trainee & Faculty \\
\hline Relevance & $\begin{array}{l}\text { Supervisors teach what is } \\
\text { relevant for the case } \\
\text { presentation. }\end{array}$ & $\begin{array}{l}\text { "I do not think anyone ever gives feedback on } \\
\text { how you spoke, on your structure ... they do } \\
\text { give feedback on what are some pertinent } \\
\text { positive or negatives that you missed,..." (trainee } \\
16 \text { (medical student)) }\end{array}$ & $\begin{array}{l}\text { "I think also the way in which they organize } \\
\text { things within those sections is really important. } \\
\text { So medications for example, [...] if it is a } \\
\text { cardiology case, I want to hear aspirin, ace } \\
\text { inhibitor, statin, all upfront, beta blocker and } \\
\text { then vitamins down below ..." (faculty 2) }\end{array}$ \\
\hline Style & $\begin{array}{l}\text { Supervisors comment on the } \\
\text { structure, pace, tone, and } \\
\text { volume of the presentation. }\end{array}$ & $\begin{array}{l}\text { "Staff like things presented differently and } \\
\text { getting feedback in terms of I would prefer it if } \\
\text { you started with this or started with the past } \\
\text { medical history first as opposed to going right } \\
\text { into the HPI ..." (trainee } 3 \text { (medical student)) }\end{array}$ & $\begin{array}{l}\text { "There is a skill to presenting that is in a way } \\
\text { like public speaking. Where the tone and the } \\
\text { pace and the emphasis is really important." } \\
\text { (faculty 2) }\end{array}$ \\
\hline Narrative & $\begin{array}{l}\text { Supervisors ensure learners stay } \\
\text { on track when presenting the } \\
\text { patient's narrative. }\end{array}$ & $\begin{array}{l}\text { "I remember my very first presentation, I gave all } \\
\text { the details. ... I remember being told by my staff: } \\
\text { the point of this is you are trying to tell a story } \\
\text { and you are trying to convince me one direction } \\
\text { or the other." (trainee } 6 \text { (PGY1)) }\end{array}$ & $\begin{array}{l}\text { "It has to make sense, there have to be no } \\
\text { surprises, and it has to be a story. When you are } \\
\text { presenting it, you have to think of your } \\
\text { conclusion first and then go back and build the } \\
\text { case so that it unfolds so that your conclusions } \\
\text { makes sense ..." (faculty } 6 \text { ) }\end{array}$ \\
\hline
\end{tabular}


"Seniors would coach the students on how to present to staff in the morning .... Usually it's sort of like seniors coaching the students or the juniors so that they don't get destroyed in the morning by the staff' (trainee 12 (PGY3)).

\section{Implicit Feedback}

A key finding that emerged from our interviews was that both trainees and supervisors had difficulty identifying the implicit feedback moments that are already richly embedded within the routine clinical activity that is the case presentation. Even though supervisors and trainees alike identified the learning culture as key to establishing an open dialogue of feedback, a hesitancy to label educational and teaching moments, like sharing clinical pearls or suggesting management changes, results in under-recognition of feedback.

\section{Interruptions as Implicit Feedback}

Supervisors were often unaware that clarifications and questions around case presentations serve as important feedback to trainees. In fact, supervisors seemed to distinguish interruptions from feedback. One explained:

"Well, when I'm interrupting them I don't probably label it as feedback, I label it as this is what I'm thinking and ... what I'm worried about, and when I hear this, this is what I'm thinking" (faculty 6)While supervisors are largely unaware of these implicit moments of feedback, trainees often perceived interruptions as a negative, rather than formative, commentary on their performance, as they felt that interruptions indicated an insufficiency in or lack of clarity of their case presentation. Trainees also analyzed their supervisors' reactions carefully, as they understood that the case presentation informed their final rotational assessment:

"You're also trying to pick out how they respond ... do they interrupt you. A lot of it is subtexts and it's very subtle. You're figuring out what questions they are asking afterwards ... you're constantly trying to evaluate through how they interact with you, whether or not you've done a quality job." (trainee 12 (PGY3))Even though many trainees were able to identify interruptions as a form of implicit feedback, a few had difficulty acknowledging this "negative" feedback as anything but punitive. As such, many trainees had difficulty labeling these moments as feedback, in fear of admitting a less than perfect performance, and a lesser evaluation. Ultimately, the desired outcome for a case presentation is one with no interruption at all, as trainees are constantly trying to impress their supervisors:
“... hopefully at the end they'll be like, "ok, that was great." Here are some things I was wondering about [ ... ]If they're happening in the middle, I don't consider that feedback, as much as I'm missing stuff' (trainee 16 (medical student))

\section{Non-verbal Behaviors}

As well, trainees expressed a keen awareness of non-verbal feedback from their clinical supervisors in determining the successful delivery of a case presentation. Trainees assigned critical meaning to these non-verbal cues, interpreting them as implicit feedback that ultimately guided their presentations and behavior. One trainee put it this way:

"I'd say maybe $20 \%$ of the time I received direct feedback on the case presentation ... and $80 \%$ of the time I had to infer ... when you're doing things that people like, they smile and nod [ ... ] [if] people don't like where you're going with things, they often interrupt or look at you curiously or blankly. It was often body language $[\ldots]$ that's how I would infer." (trainee 13 (PGY3))

\section{Feedback Culture}

Supervisors and trainees alike identified the learning culture as key to establishing an open dialogue of feedback between them. If the culture of the team was deemed supportive and formative, trainees were more comfortable presenting cases and more willing to receive feedback. However, if the environment is perceived to be unsupportive or critical, trainees were more likely to become defensive and less inclined to incorporate feedback into their clinical practice:

"If someone $[\ldots]$ is going to be interrupting me for reasons that are more critical or negative, then I do feel sort of put on the edge and I don't feel as comfortable with the presentation." (trainee 7 (PGY1))

Supervisors acknowledged these concerns, and understood the importance of feedback being provided in a respectful and supportive manner. Many supervisors used normalization as a strategy to make a trainee feel more comfortable:

" [I always try] normalizing the act of giving feedback...saying that this is formative and important part of your learning and we do it for everyone" (faculty 8 )

\section{Barriers to Feedback}

Finally, as in prior studies, participants identified a tension between the role of case presentations in providing patient care 
and as an educational activity. ${ }^{27}$ The amount of feedback provided, whether explicitly labeled or not, was limited by time constraints and supervisors balancing the "parallel tasks" (faculty 7) of digesting a patient case, while simultaneously trying to provide feedback and teach clinical pearls. ${ }^{28}$

"The challenge is that if it is a really complicated case, a lot of my attention is focused on ... . trying to figure this patient out ... I'm not as attentive to how was the organization of this, how was the resident presenting, can I hear them? Is it too fast? Is it too slow?" (faculty 2)

\section{DISCUSSION}

Our study illustrates the complex and multi-faceted nature of feedback that occurs around a case presentation. Supervisors have long struggled with the act of delivering feedback in clinical practice, and as described by Ende's (1983) classic paper, ${ }^{29}$ feedback is often omitted and handled improperly in medical education. ${ }^{30}$ Despite the commonly held belief that feedback is scarce in clinical teaching, ${ }^{30}$ our findings illustrate that in fact, feedback is occurring. Clinical supervisors understand the essential role of feedback in learner development, but are under-recognizing their behaviors and actions as representing feedback for trainees.

Although the provision of feedback is valued and prioritized by supervisors and trainees, our study illustrates that the perception of inadequate feedback likely stems from the variation in recognizing what "counts" as feedback, rather than an absolute lack of feedback. It became clear that both supervisors and trainees mainly acknowledged that feedback occurred, when it was explicitly labeled. Although some explicit feedback occurred at the time of the presentation, it usually occurred at a set point during a rotation after several case presentations had been experienced and evaluated. ${ }^{5,}{ }^{31}$ This feedback serves to inform trainees of their overall performance from a more holistic perspective. On the other hand, even though implicit feedback frequently occurred during the presentation, serving the purpose of providing contextual feedback around individual patient cases and presentations, there was less acknowledgment of this as feedback. ${ }^{5}$

As illustrated by the case presentation, trainees and supervisors hold disparate views of implicit feedback, which stems from the recognition of implicit feedback. When raising clarifications or questions during a case presentation, supervisors would often not label this as feedback. Conversely, many trainees realized that the halting of a presentation, in itself, is implicit feedback that the supervisor and trainee are not on the same page. This finding corroborates the work by Goldszmidt et al., ${ }^{28}$ where "interruptions" were described as an opportunity to clarify issues, to teach around cases, and to redirect learners. ${ }^{28}$ Our findings build on this work by demonstrating that these "interruptions" are actually representative of implicit feedback. Though not framed as feedback by supervisors, when a supervisor suggests an alternative treatment plan, adds new investigations, or elaborates their clinical reasoning, ${ }^{7}$ they are in fact providing significant implicit feedback that trainees recognize. Even further, trainees uniquely highlighted nonverbal cues from their clinical supervisors as an important component of the case presentation. They carefully analyzed their supervisors' body language and level of engagement, and incorporated this completely non-verbal, implicit feedback, to adapt their presentation content and style. ${ }^{12,}{ }^{16}$ As there is a lack of shared understanding of the role of implicit feedback, trainees may be misperceiving implicit feedback as largely negative, when it may not be intended as such.

Even more, trainees uniformly described a desire for more feedback around their case presentations. For trainees, the issue does not lie solely with the under-recognition of feedback, but also with feedback being so tightly connected with evaluation. As illustrated by our study, trainees are constantly trying to interpret and prioritize the feedback they explicitly receive, while trying to independently decode the implicit feedback that is occurring during routine clinical activities. Trainees were therefore often reluctant to label constructive feedback moments in fear of admitting criticism against their performance and ultimately the perception of a lesser evaluation. ${ }^{32,} 33$ Thus, there should be a push to improve these feedback moments, to shift the culture from one of performance to one of improvement. ${ }^{34}$

In addition, our study corroborated the literature in describing that trainees and supervisors recognized the importance of the feedback culture when providing feedback. ${ }^{35,}{ }^{36}$ Supervisors are aware of the caution required when delivering feedback, as feedback that goes against a trainee's self-assessment is not always well received, and potentially less likely to be incorporated. ${ }^{33,}{ }^{35}$ Despite avoidance of constructive feedback from supervisors, similar feedback from senior residents was highly regarded by trainees as one of the most educational aspects of their rotations. In contrast to previous studies which found medical students did not rate resident feedback highly, ${ }^{37}$ our study demonstrates that trainees readily welcome and expect constructive feedback from their senior counterparts. Near-peer feedback refines and polishes their performance, so that they feel ready for their clinical supervisors in the morning. ${ }^{38}$ Although Bordley et al. ${ }^{39}$ described residents as effective teachers, our paper is one of the first to describe the senior resident at the level of a coach providing crucial feedback on a variety of clinical activities. As learners constantly adjust their clinical roles and performance to adhere to the often unspoken principles and preferences of their supervisors, the senior resident is crucial in preparing their presentations for the morning review. ${ }^{40,}{ }^{41}$ As senior residents were often viewed as true "coaches," rather than assessors, they may provide a natural vector for the uncoupling of feedback and assessment, an elusively sought target in medical education that has proven repeatedly challenging. ${ }^{42}$ However, if senior residents have a role in high-stakes assessment of junior learners, this benefit may be lost. 
In the era of CBME, as frequent, meaningful feedback is integral, we must take advantage of the feedback already entrenched within clinical activities, like the case presentation. Implicit feedback provides trainees with contextually based, formative feedback, often without the weight of a summative performance evaluation that is often tied to explicit feedback at end of the rotation sessions. Implicit feedback is well suited for context- and task-specific feedback as it pertains to trainees' medical knowledge, understanding of relevance, ${ }^{26}$ and clinical skills. In fact, it seems to be already occurring in this form. Therefore, labeling implicit feedback for learners may help to ensure the feedback is recognized and integrated as formative, low-stakes assessment. ${ }^{35}$ Explicit feedback sessions remain important to discuss trainees' overall performance and progression. ${ }^{6,17,35}$ Our study also highlights the important role of senior residents as an additional member in the feedback relationship. Empowering senior residents to participate in feedback delivery may optimize the overall feedback culture.

Regular constructive feedback is a crucial part of CBME that should be normalized and not feared by learners, and embedded in a positive learning climate that allows for admission of limitations by the trainee. Ultimately, if supervisors understand the implications and learners properly interpret implicit feedback, the educational benefit may be two-fold: supervisors may feel more inclined to continue providing feedback, and learners may be more aware and understanding of feedback as it is occurring. Effective feedback will enable learners to refine their skills, ultimately facilitating assessment and promotion of trainees. ${ }^{13}$

\section{Limitations}

This study was conducted at a single institution with internal medicine participants. In internal medicine, the case presentation is highly valued and central to daily practice, and so our results may not be transferable to other specialties, particularly where the case presentation is shortened or less central to daily clinical practice. As well, as these case studies referred to case presentations occurring in the morning for new patients, they may not be generalizable to outpatient presentations. Further studies are needed to understand how feedback received from supervisors is integrated into trainees' future performance, especially as more and more feedback is occurring in CBME. Further work is also needed to better understand the role of senior residents as coaches and assessors in the workplace.

\section{CONCLUSION}

Our study findings illustrate the different forms and functions of feedback delivered during and after trainees' case presentations within academic Internal Medicine. Our study further describes that feedback in varying forms is occurring more frequently than supervisors acknowledge and that which has been previously described. ${ }^{30}$ Within a CBME framework, feedback holds an essential role in workplace-based assessment and promotion. This study illuminates the use of case presentations, as an exemplar of a routine clinical activity, rich with feedback, and a particular characterization of the presence and importance of implicit feedback. Therefore, utilization of routine clinical activities to maximize opportunities for delivery of feedback and other teaching activities will be critical.

Acknowledgments: We would like to thank all participants for their insights and rich commentary, and Sarah Meilach for her support.

Corresponding Author: James Rassos, MD; General Internal Medicine University of Toronto, Toronto, Ontario, Canada (e-mail: james.rassos@medportal.ca).

\section{Compliance with Ethical Standards:}

Research ethics approval was obtained from the University Health Network Research Ethics Board.

Conflict of Interest: The authors declare that they do not have a conflict of interest.

\section{REFERENCES}

1. van de Ridder JM, McGaghie WC, Stokking KM, ten Cate OT. Variables that affect the process and outcome of feedback, relevant for medical training: a meta-review. Med Educ 2015;49(7):658-73.

2. van de Ridder JM, Stokking KM, McGaghie WC, ten Cate OT. What is feedback in clinical education? Med Educ 2008;42(2):189-97.

3. Lefroy J, Watling C, Teunissen PW, Brand P. Guidelines: the do's, don'ts and don't knows of feedback for clinical education. Perspect Med Educ 2015;4(6):284-99.

4. Sender Liberman A, Liberman M, Steinert Y, McLeod P, Meterissian S. Surgery residents and attending surgeons have different perceptions of feedback. Med Teach 2005;27(5):470-2.

5. Vickery AW, Lake FR. Teaching on the run tips 10: giving feedback. Med J Aust 2005; 183(5):267-8.

6. Walsh CM, Ling SC, Wang CS, Carnahan H. Concurrent versus terminal feedback: it may be better to wait. Acad Med 2009;84(10 Suppl):S54-7.

7. Karani R, Fromme HB, Cayea D, Muller D, Schwartz A, Harris IB. How medical students learn from residents in the workplace: a qualitative study. Acad Med 2014;89(3):490-6.

8. Frank JR, Snell L, Englander R, Holmboe ES. Implementing competency-based medical education: Moving forward. Med Teach 2017;39(6):568-73.

9. Holmboe ES, Sherbino J, Long DM, Swing SR, Frank JR. The role of assessment in competency-based medical education. Med Teach 2010;32(8):676-82.

10. Duijn C, Welink LS, Mandoki M, Ten Cate OTJ, Kremer WDJ, Bok HGJ. Am I ready for it? Students' perceptions of meaningful feedback on entrustable professional activities. Perspect Med Educ 2017;6(4):256-64.

11. Chan MY. The oral case presentation: toward a performance-based rhetorical model for teaching and learning. Med. 2015;20:28565.

12. Green EH, Durning SJ, DeCherrie L, Fagan MJ, Sharpe B, Hershman W. Expectations for oral case presentations for clinical clerks: opinions of internal medicine clerkship directors. J Gen Intern Med 2009;24(3):370-3.

13. Green ML, Aagaard EM, Caverzagie KJ, Chick DA, Holmboe E, Kane $\mathbf{G}$, et al. Charting the road to competence: developmental milestones for internal medicine residency training. J Grad Med Educ 2009;1(1):5-20.

14. Frank JR, Snell L, et al. Draft CanMEDS 2015 Physician Competency Framework - Series I.Ottawa: The Royal College of Physicians and Surgeons of Canada; 2014 Feb.

15. Elnicki DM, Aiyer MK, Cannarozzi ML, Carbo A, Chelminski PR, Chheda SG, et al. An Entrustable Professional Activity (EPA)-Based Framework to Prepare Fourth-Year Medical Students for Internal Medicine Careers. J Gen Intern Med 2017;32(11):1255-60.

16. Green EH, DeCherrie L, Fagan MJ, Sharpe BA, Hershman W. The oral case presentation: what internal medicine clinician-teachers expect from clinical clerks. Teach Learn Med 2011:23(1):58-61. 
17. Watling $\mathbf{C}$, Driessen $\mathbf{E}$, van der Vleuten $\mathbf{C P}$, Vanstone $\mathbf{M}$, Lingard $\mathbf{L}$ Beyond individualism: professional culture and its influence on feedback. Med Educ 2013;47(6):585-94.

18. Dijksterhuis MG, Schuwirth LW, Braat DD, Teunissen PW, Scheele F. A qualitative study on trainees' and supervisors' perceptions of assess ment for learning in postgraduate medical education. Med Teach 2013;35(8):e1396-402.

19. Kuper A, Reeves S, Levinson W. An introduction to reading and appraising qualitative research. Bmj. 2008;337:a288.

20. Watling $\mathbf{C J}$, Lingard $\mathbf{L}$. Grounded theory in medical education research: AMEE Guide No. 70. Med Teach 2012;34(10):850-61.

21. Melvin L, Rassos J, Panisko D, Driessen E, Kulasegaram KM, Kuper A. Overshadowed by Assessment: Understanding Trainee and Supervisor Perspectives on the Oral Case Presentation in Internal Medicine Workplace-Based Assessment. Acad Med 2019;94(2):244-50.

22. Charmaz K, Belgrave L. Qualitative interviewing and grounded theory analysis. The SAGE handbook of interview research: The complexity of the craft 2002;2:2002.

23. Charmaz K. The power and potential of grounded theory. Medical Sociology Online 2012;6(3):2-15.

24. Morse JM. Critical Analysis of Strategies for Determining Rigor in Qualitative Inquiry. Qual Health Res 2015;25(9):1212-22.

25. Hennink MM, Kaiser BN, Marconi VC. Code Saturation Versus Meaning Saturation: How Many Interviews Are Enough? Qual Health Res 2017;27(4):591-608.

26. Lingard LA, Haber RJ. What do we mean by "relevance"? A clinical and rhetorical definition with implications for teaching and learning the casepresentation format. Acad Med 1999;74(10 Suppl):S124-7.

27. Reddy ST, Zegarek MH, Fromme HB, Ryan MS, Schumann SA, Harris IB. Barriers and Facilitators to Effective Feedback: A Qualitative Analysis of Data From Multispecialty Resident Focus Groups. J Grad Med Educ 2015;7(2):214-9.

28. Goldszmidt $\mathbf{M}$, Aziz N, Lingard $\mathbf{L}$. Taking a detour: positive and negative effects of supervisors' interruptions during admission case review discussions. Acad Med 2012;87(10):1382-8.

29. Ende J. Feedback in clinical medical education. Jama. 1983;250(6):777-81.

30. Peter A. M. Anderson. Giving Feedback on Clinical Skills: Are We Starving Our Young? J Grad Med Educ 2012;4(2):154-8.
31. Grierson LE. We're talking about feedback... aren't we? Adv Health Sci Educ Theory Pract 2012;17(1): 1-4

32. Ramani S, Post SE, Konings K, Mann K, Katz JT, van der Vleuten C. "It's Just Not the Culture": A Qualitative Study Exploring Residents' Perceptions of the Impact of Institutional Culture on Feedback. Teach Learn Med 2016:1-9.

33. Mann K, van der Vleuten C, Eva K, Armson H, Chesluk B, Dornan T, et al. Tensions in informed self-assessment: how the desire for feedback and reticence to collect and use it can conflict. Acad Med 2011;86(9):1120-7.

34. Watling CJ, Ginsburg S. Assessment, feedback and the alchemy of learning. Medical education. 2019;53(1):76-85.

35. Watling $\mathbf{C}$, Driessen $\mathbf{E}$, van der Vleuten $\mathbf{C P}$, Lingard $\mathbf{L}$. Learning culture and feedback: an international study of medical athletes and musicians. Med Educ 2014;48(7):713-23.

36. Ross S, Dudek N, Halman S, Humphrey-Murto S. Context, time, and building relationships: bringing in situ feedback into the conversation. Med Educ 2016;50(9):893-5

37. Melvin L, Kassam Z, Burke A, Wasi P, Neary J. What Makes a Great Resident Teacher? A Multicenter Survey of Medical Students Attending an Internal Medicine Conference. J Grad Med Educ 2014;6(4):694-7.

38. LaDonna KA, Hatala $\mathbf{R}$, Lingard L, Voyer S, Watling C. Staging a performance: learners' perceptions about direct observation during residency. Medical education. 2017;51(5):498-510.

39. Bordley DR, Litzelman DK. Preparing residents to become more effective teachers: a priority for internal medicine. Am J Med 2000;109(8):693-6.

40. Apramian T, Cristancho S, Watling C, Ott M, Lingard L. Thresholds of Principle and Preference: Exploring Procedural Variation in Postgraduate Surgical Education. Acad Med 2015;90(11 Suppl):S70-6.

41. Sargeant J, Eva KW, Armson H, Chesluk B, Dornan T, Holmboe E, et al. Features of assessment learners use to make informed selfassessments of clinical performance. Med Educ 2011;45(6):636-47.

42. Cavalcanti RB, Detsky AS. The education and training of future physicians: why coaches can’t be judges. Jama. 2011;306(9):993-4.

Publisher's Note Springer Nature remains neutral with regard to jurisdictional claims in published maps and institutional affiliations. 